\title{
The effects of pronoun processing on information utilization during fixations in reading
}

\author{
HARRY E. BLANCHARD \\ University of Illinois at Urbana-Champaign, Urbana, Illinois
}

(Keith Rayner, Sponsor)

\begin{abstract}
This experiment tested the hypothesis that the time of utilization of information during eye fixations is sometimes delayed because concurrent comprehension processes are still occupied with analyzing previous information (the variable utilization time hypothesis). The method of determining the time of utilization used in previous experiments (Blanchard, McConkie, Zola, \& Wolverton, 1984) was combined with a manipulation of comprehension difficulty. The comprehension manipulation involved varying the distance between a pronoun and its referent, which Ehrlich and Rayner (1983) had shown to cause delayed processing effects. The desired effects were not obtained in this experiment; thus the variable utilization time hypothesis cannot be properly tested with these results. Reasons for the failure to replicate Ehrlich and Rayner's (1983) effects are investigated. Also, some unpredicted results that support the variable utilization time hypothesis are discussed.
\end{abstract}

In reading, the eye makes a series of saccades from one point in the text to another. Between the saccades are fixations, during which the useful visual information for reading is acquired. During the entire period of fixation, visual information is made available to the brain. At some point, visual information is passed on to higher level processors in order to further comprehension of the text. This point in time is called the utilization of visual information (and is distinguished from simple registration, which is transmission of information to the visual cortex and perhaps some early pattern recognition; see McConkie, 1983).

Earlier experiments (Blanchard, 1985; Blanchard, McConkie, Zola, \& Wolverton, 1984) showed that visual information is not utilized at the same point in every fixation. Visual information is sometimes utilized early in a fixation and sometimes late. In these experiments, subjects read from a computer screen while their eye movements were monitored. A single letter (the critical letter) was changed partway through each fixation. For each text the subjects read, the critical letters resided in two critical words that both fit in the text: for example, The underground caverns were meant to house hidden (tombs, bombs), but then the construction was stopped because of lack of funds. On fixations near the critical word, the word initially present, tombs, was changed to its alternative, bombs, after some delay (50-120 msec). During saccades, the initially presented word returned. In addition, a 30-msec mask was presented to eliminate localized apparent movement caused by changing the critical letter.

The result was that subjects frequently reported seeing only one of the critical words. They sometimes reported

\footnotetext{
Support for this research came from National Institute of Education Contract 400-81-0030 to the Center for the Study of Reading, University of Ilinois. Requests for reprints should be sent to Harry Blanchard, Department of Psychology, University of Massachusetts, Amherst, MA 01003.
}

the first word, sometimes the second, and sometimes both words. If information is always utilized immediately after registration, then subjects should always consistently report the first word in these experiments. Clearly, information is not always utilized immediately after the completion of early visual processing. Sometimes there is a delay, in which visual information remains available but is not utilized until much later in the fixation.

The corollary of this conclusion is that something other than the completion of registration determines when information is utilized by comprehension processes. Blanchard et al. (1984) introduced this concept as the variable utilization time hypothesis and claimed that the time of utilization is sometimes early and sometimes late in the fixation. The reason why utilization is sometimes delayed is that the comprehension processes are not ready to accept new visual information because they are occupied with analyzing previous information. Only when the comprehension processes are in a state where comprehension can be furthered by new visual information does utilization occur.

This experiment explicitly tested the variable utilization time hypothesis. The changing-letter procedure used by Blanchard et al. (1984) was used here, combined with a manipulation that is known to affect comprehension difficulty. The goal was to use a manipulation of comprehension processes to cause selective changes in the probability of reporting the first and second of the changing words.

The comprehension process manipulation chosen was the difficulty of the semantic integration of a pronoun preceding the critical word. The referent of the pronoun appeared either immediately before the pronoun (near condition) or one to three sentences prior to the pronoun ( far condition; see Table 1). This manipulation was used 
Table 1

Sample Text in Two Pronoun-Referent Distance Conditions

\section{Near Condition}

This was clearly another act of the Hillsdale Strangler, which was why Farnsworth was brought in on the case. Detective Harold Farnsworth looked at the murdered woman. Her (plaid, plain) blouse was splattered with blood. But an insignia on the blouse linked the woman to a Satanic cult.

\section{Far Condition}

Detective Harold Farnsworth looked at the murdered woman. This was clearly another act of the Hillsdale Strangler, which was why Farnsworth was brought in on the case. Her (plain, plaid) blouse was splattered with blood. But an insignia on the blouse linked the woman to a Satanic cult.

by Ehrlich (1983) and Ehrlich and Rayner (1983) in eye movement experiments that did not involve any text changes on the screen. They found that in the near condition semantic integration of the pronoun was completed on the fixation on which the pronoun was visually acquired, and that in the far condition semantic integration was not completed until one to two fixations after the pronoun was acquired. This was indicated by the pattern of fixation durations in the vicinity of the pronoun.

The delayed processing effects suggest that there should be spillover of pronoun processing on the fixation following pronoun encoding, where, in this study, the critical word was located. The predictions from the variable utilization time hypothesis are as follows. In the near condition, pronoun assignment is easy. Pronoun processing should usually be completed on the pronoun-encoding fixation. When the eye is on the critical word location, information should be utilized early during the fixation, because comprehension processes are ready to accept new visual input immediately. Hence, the first word should be reported more often. In the far condition, pronoun assignment is more difficult than in the near condition, and pronoun processing should extend beyond the pronounencoding fixation (as found by Ehrlich \& Rayner, 1983). When the eye is on the critical word location, comprehension processes may not be ready to integrate new information until pronoun processing is completed. Thus utilization during the fixation on the changing word should be more likely to occur late in the fixation; hence, the second word should be reported more often.

\section{METHOD}

\section{Subjects}

Sixteen college students or graduates with normal uncorrected vision, who were native speakers of English, were subjects in this experiment.

\footnotetext{
Apparatus

The text was displayed one line at a time, in upper- and lowercase letters on a DEC VT-11 display unit. The subjects were $68 \mathrm{~cm}$ from the display, and $i^{\circ}$ of visual angle encompassed four character positions. The subjects read the passages by pressing a button each time they needed to go forward to a new line. While subjects were reading, their eye movements were recorded every millisecond with an SRI Dual Purkinje Image Eyetracker in conjunction with a DEC PDP-11/40 minicomputer. Head movements were reduced by a headrest and bitebar.
}

\section{Materials}

Each three- to five-sentence text had two different versions, which were different in meaning but visually different by only one letter. This letter was located in a five-letter critical word (in any of the five letter positions). In addition, the texts were written so as to contain the Ehrlich and Rayner (1983) pronoun-referent distance manipulation. Each critical word immediately followed a pronoun. The pronoun was the first word in a sentence. In the near condition the referent immediately preceded the pronoun, whereas in the far condition the referent was one to three sentences before the pronoun. An example is shown in Table 1, and a complete listing appears in Blanchard (1985).

\section{Procedure}

On every fixation subjects made in reading the texts, the text was momentarily masked for $30 \mathrm{msec}$ at some time during the fixation. (The mask consisted of two overprinted lines of uppercase Xs and Os overprinted at each character position, with one line extending below the text line.) The time between the beginning of a fixation and the onset of the mask was either 50 or $100 \mathrm{msec}$. Thus each fixation was divided into three time periods: the time before the mask (either 50 or $100 \mathrm{msec}$ ), mask time (always $30 \mathrm{msec}$ ), and the time after the mask (variable, depending on the fixation duration).

In the experimental condition, the letter that distinguished the two alternate versions of the text was changed during certain selected fixations, whereas in the control condition, this critical letter was not changed (the line of text that reappeared after the mask was identical to the line that appeared before the mask). The letter changed in the critical word only if (1) at least one fixation had previously occurred within 12 character positions of the left end of the line, (2) the fixation was located within a region extending 11 character positions to the left and right of the first letter of the critical word, and (3) since initially entering this region, the eye did not pass out of it to the right (i.e., no changes on regressions aimed back into the region from outside the region).

Assignment of texts to conditions was random and counterbalanced, and presentation order of critical words in the fixation was counterbalanced across subjects. Subjects were informed about the mask and the changing letters but not about the pronoun-referent distance manipulation.

A recognition test procedure was used to determine which word(s) subjects had read. After the subject had read a text, four test words appeared individually, one after the other. For each word, the subject identified whether it had appeared in the immediately preceding text by pressing a "yes" or "no" button. Two of the test words were the critical words. The other two test words also differed by one letter, although one of these words never appeared in the text. Presentation order of test words was randomized.

\section{RESULTS AND DISCUSSION}

To provide a test of the variable utilization time hypothesis, it was necessary to determine whether this experiment successfully obtained the pronoun processing effects found by Ehrlich and Rayner (1983). The algorithm used by Ehrlich and Rayner was used to select four fixation sets: the fixation prior to encoding the pronoun, the fixation on which the pronoun was encoded, the fixation following encoding, and the second fixation after encoding. The algorithm defined the pronoun-encoding fixation as the fixation that was closest to the pronoun and that fell within a region extending six character positions to the left of the first letter of the pronoun and one character position to the right of the final letter of the pronoun. In the current experiment, fixations following encoding were likely to fall on the critical changing word. The data from the control condition of the present experiment were compared to Ehrlich and Rayner's (1983) data from the near and far conditions of their Experiment 2. 
Table 2

Mean Durations of Fixations at Various Locations Around the Pronoun

\begin{tabular}{lcccc}
\hline \multicolumn{1}{c}{ Distance } & \multicolumn{4}{c}{ Fixation } \\
\cline { 2 - 5 } & Before & Encoding & One After & Two After \\
\hline $\begin{array}{l}\text { Ehrlich \& Rayner (1983) } \\
\text { Near }\end{array}$ & 224 & 248 & 224 & 207 \\
$\quad$ Far & 220 & 242 & 269 & 296 \\
This Experiment & & & & \\
$\quad$ Near & 247 & 221 & 245 & 245 \\
Far & 224 & 199 & 250 & 234 \\
No Mask or Changing Letters & & & & \\
Near & 227 & 208 & 228 & 208 \\
Far & 217 & 190 & 243 & 204 \\
He and She Only & & & & \\
Near & 237 & 223 & 232 & 246 \\
Far & 234 & 223 & 261 & 242 \\
\hline
\end{tabular}

Note-Fixation durations are in milliseconds.

Table 2 presents the mean fixation durations for the four fixation sets defined by the above algorithm. The pattern of effects is different from Ehrlich and Rayner's (1983) results. In particular, there was no increase in the duration of fixations following encoding of the pronoun in the far condition relative to that in the near condition. This is the effect that Ehrlich and Rayner (1983) attributed to delayed processing of the pronoun, and that is crucial to producing the effects predicted in this experiment. In a repeated measures analysis of variance, the main effects of pronoun-referent distance $[F(1,15)=4.80$, $p=.05]$ and serial order of fixation $[F(3,45)=9.36$, $p \cong 0]$ were significant. The interaction was not significant.

The failure to obtain the delayed processing effects reported by Ehrlich and Rayner (1983) could be due to some effect of the midfixation masking on language processing. To examine this possibility, a second experiment was conducted with the same texts, but with no masking and no changing letters. Eighteen subjects, different from those used in the main experiment, read the texts and performed the recognition tests with the same test words used in the main experiment. The effect of pronoun-referent distance on mean fixation durations was examined in the same way as in the main experiment. There was an increase in mean fixation duration in the far condition relative to that in the near condition on the first fixation after pronoun encoding (Table 2), but not on the second fixation after pronoun encoding. The pattern of fixation duration effects does not match the results of Ehrlich and Rayner (1983) but is similar to the pattern in the main experiment. The main effect of pronoun-referent distance was not significant, but serial order of fixation was significant $[F(3,51)=16.10, p \cong 0]$, as was the interaction $[F(3,50)=5.40, p=.003]$. In sum, the mask presented during each fixation in the main experiment cannot account for the difference in results between that experiment and the original Ehrlich and Rayner (1983) experiments.

The most likely explanation for the different results of these two experiments lies in some linguistic property of the texts. One possibility is that the current experiment used other pronouns in addition to the pronouns he and she. At least some of these other pronouns might have had different effects on ongoing language processes. To examine this possibility, the effect of pronoun-referent distance on fixation durations (in the main experiment) was analyzed for only those texts in which he and she were used as the critical pronouns. There was an increase in mean fixation duration for the first fixations after pronoun encoding, but no increase for the second fixations after encoding. Furthermore, neither the main effects of distance and serial order of fixation nor the interaction were significant. Thus the difference in pronouns cannot be the major explanation for the difference between this experiment and that of Ehrlich and Rayner (1983).

The difference between the current texts and those of Ehrlich and Rayner (1983) may be in the discourse structure of the texts in the far condition. Ehrlich and Rayner gave an example of one of their texts in the far condition:

A group of people who shared an interest in photography had recently started writing a newsletter of their activities. Mark wrote most of the copy but the other members did a lot of work as well. In fact, in one room Cathy was mailing a copy of the paper to Susan. He was very involved in photography and spent every weekend taking pictures. (p. 80, Table 1; italics added)

Between the pronoun he and its referent Mark, the focus or topic is changed from Mark to Cathy and Susan. The reader has no expectation that the discourse will return to Mark, but, at the pronoun he, it does. In the present experiment, the text intervening between the pronoun and its referent in the far version (see Table 1) does not change focus and does lead the reader to expect more information about the woman later in the text. Most of the texts in this experiment are constructed similarly, as the result of an effort to keep the near and far texts as similar as possible.

The significance of this difference depends on what kind of linguistic structure is actually responsible for increased processing time required by a pronoun. It may simply be 
Table 3

Percentage of Single-Word Reports in Two Experiments

\begin{tabular}{lcc}
\hline & \multicolumn{2}{c}{ Word Reported } \\
\cline { 2 - 3 } Experiment & First & Second \\
\hline Blanchard et al. (1984) & 45 & 55 \\
This Experiment & 64 & 36 \\
\hline
\end{tabular}

the number of words between a pronoun and its referent. However, it may be a sort of "mental distance" that is important: the referent to a pronoun becomes harder to compute as that referent is further in the background of the current mental representation of the text. This would be correlated with physical distance, but physical distance would not be sufficient to move a referent out of the current focus of the discourse. Kantor (1977), Carroll and Slowiaczek (in press), and Clifton and Ferreira (in press) have made similar arguments, and the latter have reported data consistent with these ideas.

Given that the materials used in this experiment failed to reproduce the delayed processing effects in the far condition found by Ehrlich and Rayner (1983), this experiment does not provide an adequate test of the predictions of the variable utilization time hypothesis as presented earlier. However, a post hoc interpretation of this study is possible if the above argument about discourse focus and pronoun processing is correct. If it is, then in the current study pronoun processing may have been facilitated in both the near and far conditions. According to the original predictions, easy pronoun processing allows early utilization of the changing word. Therefore, utilization may tend to occur early on fixations in both the near and far conditions. However, to test this, some neutral comparison is needed. The best available comparison is the pattern observed in Blanchard et al. (1984). Consistent with this new prediction, in the present study, the word presented first in the fixations was reported more often than the second word, unlike in Blanchard et al.'s (1984) study, in which the probabilities of reporting the first or the second word were nearly equal. Table 3 presents the percentage of single-word reports that were reports of the first and second words in this experiment and in Blanchard et al.'s (1984) study. Only the current experiment shows a significant difference by a chi-square test on the frequencies for first and second word reported $\left[\chi^{2}(1\right.$, $N=16)=17.63, p<.001]$.

Firm conclusions cannot be made from this experiment, because the desired pronoun-processing effects were not produced. However, there is some suggestion by post hoc analysis that the variable utilization time hypothesis is supported. Further research is needed to confirm this. Another implication of this study is that the difficulty of semantic integration of a pronoun is a function not of the distance between the pronoun and its referent, but rather of discourse characteristics of the text, namely, whether the referent of the pronoun has been kept in the foreground or background of the ongoing mental representation of the text.

\section{REFERENCES}

BLANChARD, H. E. (1985). The dynamics of perception during fixations in reading. Unpublished doctoral dissertation, University of IIlinois, Urbana-Champaign.

BlanchaRd, H. E., McConkie, G. W., Zola, D., \& Wolverton, G. (1984). Time course of visual information utilization during fixations in reading. Journal of Experimental Psychology: Human Perception \& Performance, 10, 75-89.

Carroll, P., \& Slowiaczex, M. L. (in press). Modes and modules: Multiple pathways in the language processor. In J. L. Garfield (Ed.), Modularity in sentence comprehension: Knowledge representation and natural language understanding. Cambridge, MA: MIT Press.

Clufton, C., JR., \& Ferreira, F. (in press). Discourse structure and anaphora: Some experimental results. In M. Coltheart (Ed.), Attention and performance XII. Hillsdale, NJ: Erlbaum.

EHRLICH, K. (1983). Eye movements in pronoun assignment: A study of sentence integration. In K. Rayner (Ed.), Eye movements in reading: Perceptual and language processes (pp. 253-268). New York: Academic Press.

EhrLich, K., \& RAYNER, K. (1983). Pronoun assignment and semantic integration during reading: Eye movements and immediacy of processing. Journal of Verbal Learning \& Verbal Behavior, 22, 75-87.

KANTOR, R. N. (1977). The management and comprehension of discourse connection by pronouns in English. Unpublished doctoral dissertation, Ohio State University, Columbus.

MCCONKIE, G. W. (1983). Eye movements and perception during reading. In K. Rayner (Ed.), Eye movements in reading: Perceptual and language processes (pp. 65-96). New York: Academic Press.

(Manuscript received for publication November 24, 1986.) 